Original

\title{
Valor de la angiogénesis en relación a otros factores morfológicos en el carcinoma ductal infiltrante de mama
}

F. J. Torres Gómezi ${ }^{1}$ F. J. Torres Olivera²

\section{Resumen}

Introducción: Diferentes experiencias sugieren que el carcinoma ductal infiltrante es un tumor dependiente de la angiogénesis para adquirir capacidad invasora y metastásica. El valor de la angiogénesis como factor pronóstico suscita opiniones encontradas. Diversos factores se han invocado como responsables de las discrepancias en los resultados. Hemos correlacionado la angiogénesis con otros factores pronósticos consensuados, a fin de establecer su posible valor pronóstico.

Material y métodos: En 185 pacientes con carcinoma ductal infiltrante (NOS) hemos determinado la densidad vascular con un método de recuento estandarizado y la hemos relacionado con el tamaño tumoral, grado histológico, estatus ganglionar axilar, número de ganglios afectados y con cada uno de los parámetros que integran el grado histológico (formación tubular, grado nuclear e índice mitósico).

Resultados y conclusiones: Hemos obtenido correlación estadísticamente significativa entre la densidad vascular (DV) y los parámetros considerados con anterioridad.

Palabras clave: Angiogénesis. Carcinoma ductal infiltrante. Mama. Tamaño tumoral. Grado histológico, estatus ganglionar.

Oncología, 2007; 30 (3):92-99

${ }^{1}$ Servicio de Anatomía Patológica. Hospital Punta de Europa. Algeciras (Cádiz)

2 Departamento de Anatomía Patológica. Hospital Universita- 


\section{Summary}

Introduction: Different experiences suggest that breast invasive ductal carcinoma depends on angiogenesis to reach invasive and metastatic properties. The value of angiogenesis as a prognostic factor encounters conflicting opinions. We studied the correlation between angiogenesis and other accepted prognostic factors in order to assess its prognostic value.

Material and methods: We calculated the vascular density of 185 patients with breast invasive ductal carcinoma using a standardized counting method to asses the correlation of angiogenesis with tumour size, histologic grade, axillary lymph nodes state, and the different features of the histological grade (tubular formation, nuclear grade, and mitotic index).

Results and conclusions: We found significant statistic correlation between vascular density and the aforementioned items.

Key words: Angiogenesis. Breast. Invasive ductal carcinoma. Tumour size. Histological grade. Lymph node sate.

\section{Introducción}

La angiogénesis es un proceso necesario para que un tumor maligno adquiera potencial invasor y metastásico ${ }^{1-6}$ y para el crecimiento del depósito metastásico en el órgano diana y su posterior proceso de diseminación ${ }^{6}$. Numerosas experiencias sostienen que el cáncer mamario es un tumor dependiente de la angiogénesis ${ }^{4-11}$. La resonancia magnética con contraste encuentra una relación evidente entre el incremento de densidad vascular (DV) y aparición de cáncer mamario ${ }^{12}$. La expresión de angiogénesis es precoz y detectable en el estadio intraductal ${ }^{13-15}$, observándose incremento de la DV alrededor de los ductos afectados en relación a la observada en ductos normales ${ }^{15}$. Asimismo, la DV observada alrededor de ductos con comedocarcinoma (un fenotipo de mayor agresividad biológica) es mayor que la observada alrededor de ductos con carcinoma intraductal habitual ${ }^{15}$ y también está aumentada en los márgenes infiltrativos del carcinoma mamario $5,6,16,17$. El aumento de la DV es un indicador pronóstico significativo independiente del estatus ganglionar axilar ${ }^{4,5,6,18-23}$, se relaciona con mayor frecuencia de metástasis ${ }^{24}$, con un número superior de ganglios afectados ${ }^{25} \mathrm{y}$ con menor supervivencia ${ }^{21,26}$. Se ha establecido una relación directa entre la DV intratumoral y el riesgo de metástasis en el carcinoma de ma$\mathrm{ma}^{5,6,27,28}$. Todos estos hechos sugieren la validez de la angiogénesis como factor pronóstico en el carcinoma mamario $0^{5,6,9,21,23,25-30}$ ya establecida en el melanoma ${ }^{31}$ y en otros órganos ${ }^{32,33}$.

Otros autores encuentran relación entre DV y grado histológico tumoral, pero no con otros factores pronósticos clásicos (tamaño tumoral y estatus ganglionar) $)^{34,35,37-39} \mathrm{ni}$ con la supervivencia ${ }^{40}$.

La utilización de diferentes marcadores vasculares $^{41}$, la heterogeneidad de los tipos histológicos tumorales estudiados ${ }^{42,43}$, los diferentes métodos de elección de las áreas de mayor $\mathrm{DV}^{6,17,44,45}$ junto a la subjetividad y variaciones interobservadores $6,29,37-39$ contribuyen sin duda a estas importantes discrepancias.

En un estudio anterior ${ }^{46}$ hemos tratado de estandarizar las técnicas de marcado y recuento vascular, la evaluación de las áreas de mayor DV (puntos calientes) y la homogenización del tipo histológico a valorar, con el fin de obtener una reproducibilidad aceptable de los resultados ${ }^{47,48}$. En el presente trabajo hemos correlacionado la DV obtenida bajo esas premisas, con los factores pronósticos morfológicos ampliamente concensuados en el carcinoma de mama, a fin de establecer si la angiogénesis puede ser considerada como un factor pronóstico más en este tipo de neoplasia. 


\section{Material y métodos}

Hemos estudiado 332 especimenes de tejido mamario y linfadenectomías axilares de 189 pacientes remitidas al Departamento de Anatomía Patológica del Hospital Universitario Virgen Macarena de Sevilla entre los años 1996 y 2004. Se han estudiado exclusivamente los carcinomas ductales infiltrantes clásicos que no hubieran recibido tratamiento radioterápico o quimioterápico previos. Una vez fijadas las piezas en formol acuoso al $10 \%$ durante 24 horas se ha procedido a medir el diámetro máximo tumoral y a obtener secciones representativas del tumor que incluyeran el borde de crecimiento. Tras la inclusión en parafina en un procesador automático, se han obtenido los bloques de los que a su vez se obtuvieron cortes histológicos de 3 micras de espesor que se tiñeron con Hematoxilina-Eosina (HE). De cada caso se obtuvieron cortes histológicos montados sobre portas cargados eléctricamente destinados a estudio inmunohistoquímico, utilizando el anticuerpo correspondiente frente a CD31 (monoclonal, dilución 1:50, Concepta Byosystems S.A., Barcelona) en un procesador automático VENTANA ES (Ventana Medial Systems, Tucson, Arizona, USA), empleando el conjunto de detección Ventana Enhanced DAD (solución inhibidora de peróxido de hidrógeno, anticuerpo biotinilado antiratón, solución de peróxido de hidrógeno, DAD, solución de sulfato de cobre, hematoxilina y fondo azuleante). El desenmascaramiento antigénico se ha realizado mediante tampón citrato a $\mathrm{Ph} 6$, en olla a presión durante 4 minutos. El estudio microscópico de ha realizado en un microscopio Olimpos BX 40 (Tokio, Japón).

Sobre los cortes histológicos teñidos con HE se ha establecido el grado histológico combinado de Nottingham ${ }^{48}$. La valoración de la DV se ha establecido según el protocolo descrito anteriormente ${ }^{46} \mathrm{y}$ se ha correlacionado con el tamaño tumoral (TT), tamaño tumoral medio (TTM), grado histológico combinado $(\mathrm{GH})$ y sus parámetros (formación tubular (FT), grado nuclear (GN) e índice mitósico (IM), estatus ganglionar axilar (EG) y número de ganglios axilares afectados. La obtención de la DV se ha realizado sin conocer previamente el GH ni el EG.

El análisis estadístico se ha realizado con el programa SPSS versión 12.0 para Windows. Los métodos utilizados han sido: a) en el caso de análisis de variables cualitativas o cuantitativas agrupadas en intervalos, se han calculado las Tablas de Contingencia u Homogeneidad con el Test de la Chi-cuadrado o en el caso de Tablas 2 x 2 con la corrección de continuidad; b) en el caso de comparación de variables según los grupos que establece una variable cualitativa, hemos utilizado el Análisis de la Varianza (ANOVA) y en el supuesto de obtener diferencias significativas hemos utilizado el Test de Comparaciones Múltiples de Bonferroni.

\section{Resultados}

La edad media de las pacientes presenta un rango entre los 32 y 85 años (edad media 58,49 años). La mayor incidencia se encuentra en la década de los 50 (55 casos, 29,10\%). La afectación de la mama izquierda (104 casos, $55,03 \%$ ) es ligeramente superior a la de la derecha (85 casos, $44,97 \%$ ).

El tamaño tumoral (TT) osciló entre 0,50 y 13 cms (tamaño tumoral medio (TTM) 2,65 cms. En 7 casos $(3,70 \%)$ el TT ha sido inferior a $1 \mathrm{~cm}$. El grupo más numeroso corresponde a las lesiones de tamaño comprendido entre 1 y $2 \mathrm{cms}$ (87 casos, $46,03 \%$ ), siendo casi equiparables los grupos de lesiones de tamaños comprendidos entre los 2 y $3 \mathrm{cms}$ y los mayores de $3 \mathrm{cms}$ (49 y 48 casos respectivamente).

20 casos $(10,58 \%)$ mostraron buena diferenciación tubular (FT1), $44(23,28 \%)$ moderada (FT2) y $125(66,14 \%)$ escasa o nula (FT3).

41 tumores $(21,69 \%)$ mostraron grado nuclear bajo (GN1), $103(54,50 \%)$ moderado (GN2) y 45 $23,81 \%)$ alto $(\mathrm{GN} 3)$.

95 casos $(50,26 \%)$ mostraron baja actividad mitósica (IM1), $45(23,81 \%)$ moderada (IM2) y 49 $(25,93 \%)$ alta (IM3)

La densidad vascular total (DVT) obtenida en los 189 casos sobre el mejor punto caliente, en un campo microscópico a $400 \mathrm{x}$, ha sido de 8112 vasos, y la densidad vascular media (DVM) de 42,92 vasos.

La DVM obtenida en los tumores con TT hasta 2 cms es menor que las obtenidas en carcinomas de TT superior a $2 \mathrm{cms}$ (Fig. 1). Cuando las DV se agrupan por intervalos se aprecia un predominio de los casos con TT superior a $2 \mathrm{cms}$ a partir de un recuento vascular de 60 vasos (Fig. 2).

La DVM obtenida en los casos con FT1 es sen- 


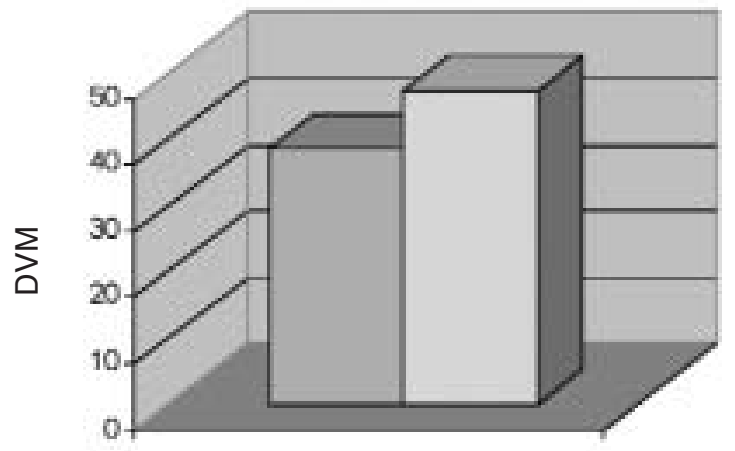

tamaño tumoral

a $0.2 \mathrm{cms}$ a $>2 \mathrm{cms}$

Fig. 1. Correlación entre TT y DV.

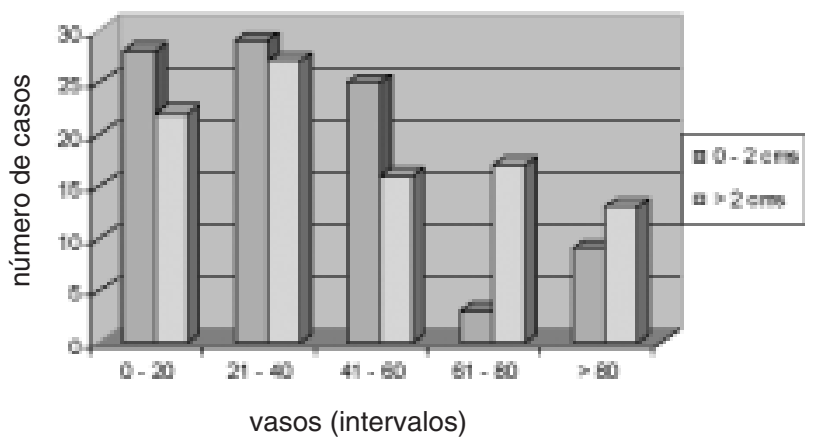

Fig. 2. Correlación entre TT y DV.

siblemente menor que las obtenidas en los casos con FT2 y FT3 (Fig. 3). Estas diferencias se mantienen cuando los recuentos se agrupan en intervalos (Fig. 4).

Las DVM obtenidas en los carcinomas con GN2 y GN3 son mayores que las obtenidas en los casos con GN1 (Fig. 5). Asimismo dichas diferencias se mantienen al agrupar los recuentos vasculares por intervalos (Fig. 6).

La DVM obtenida en los carcinomas con IM1 es menor que las obtenidas en los tumores con IM2 e IM3 (Fig. 7). Dichas diferencias se mantienen al agrupar los recuentos vasculares por intervalos (Fig. 8).

La DVM se incrementa en relación directa con el aumento del GH (Fig. 9). Esta relación se mantiene cuando se agrupan los recuentos vasculares por intervalos (Fig. 10).

La DVM obtenida en los tumores con ganglios

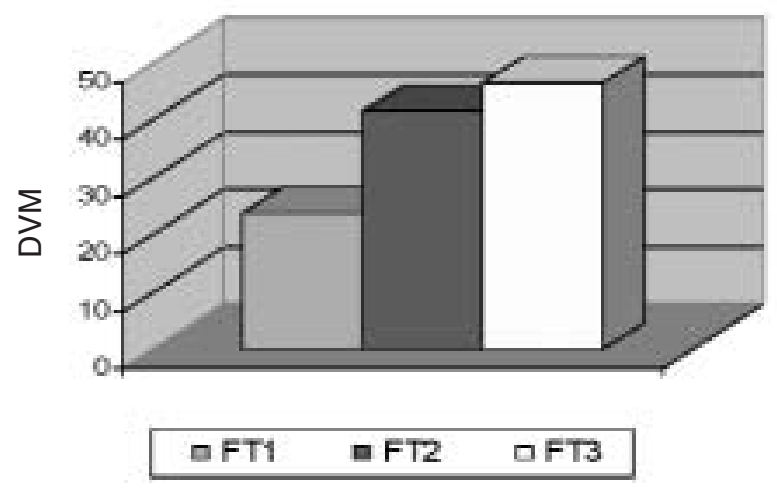

Fig. 3. Correlación entre FT y DV.

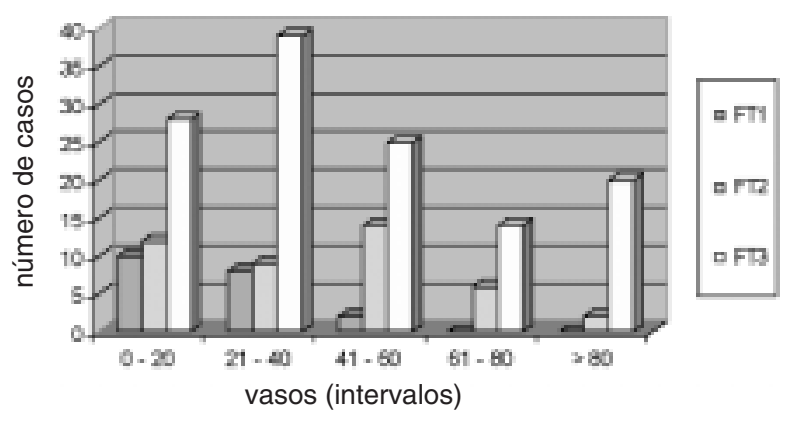

Fig. 4. Correlación entre FT y DV.

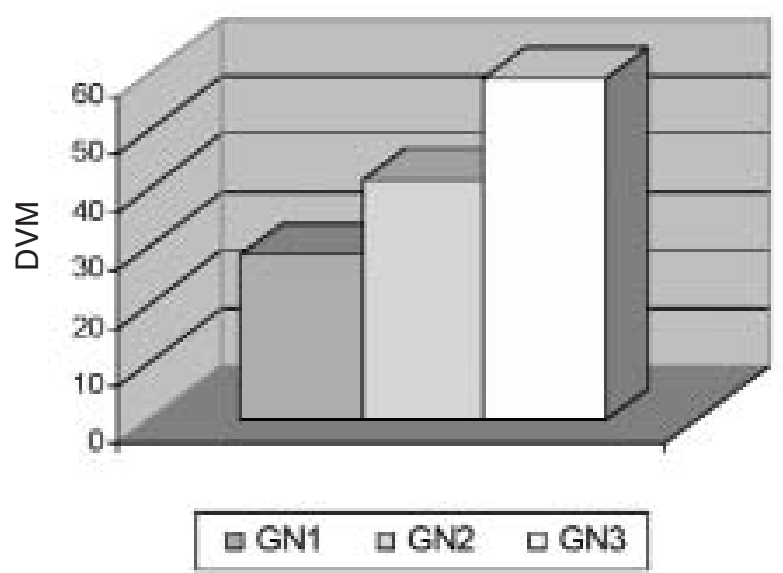

Fig. 5. Correlación entre GN y DV.

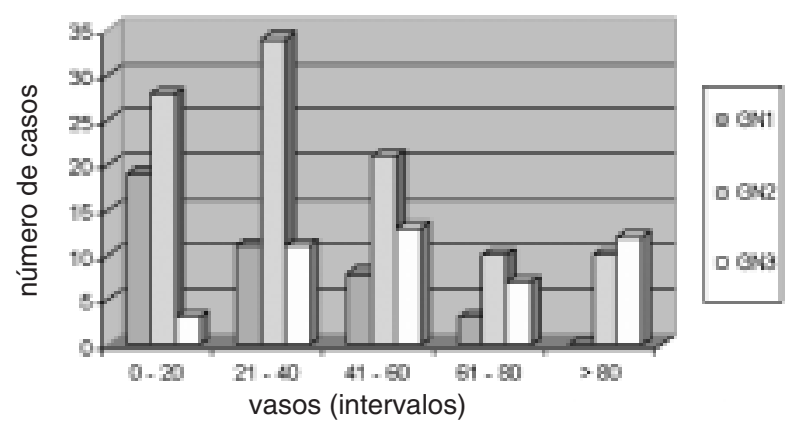

Fig. 6. Correlación entre GN y DV. 


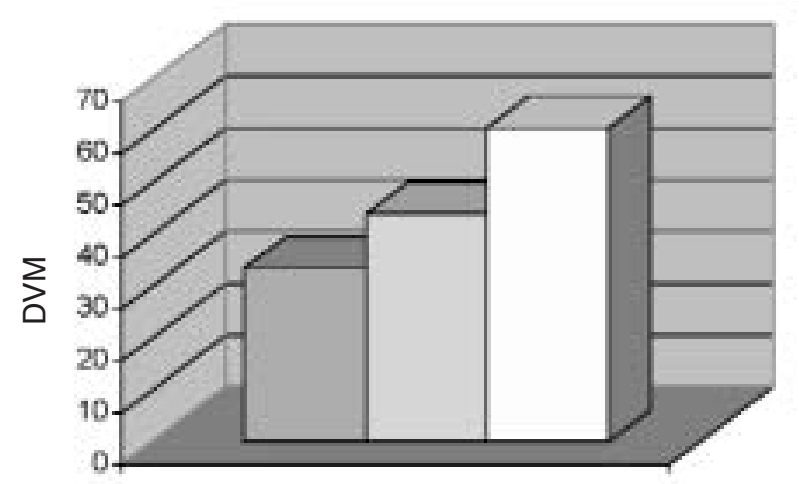

\section{ㅁI1 면 면}

Fig. 7. Correlación entre IM y DV.

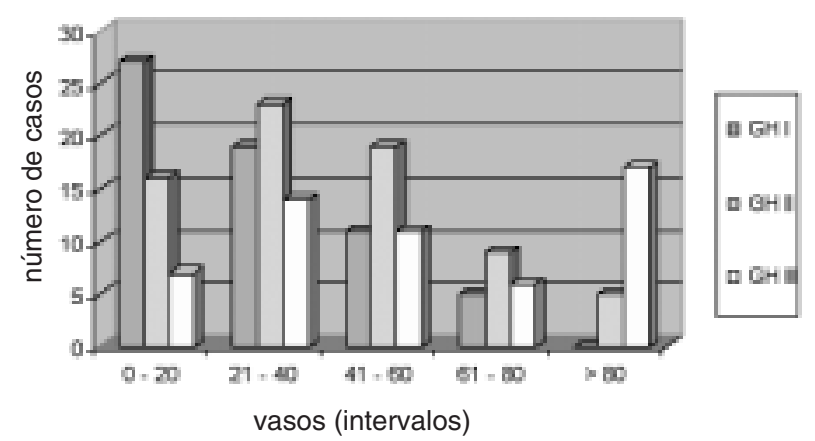

Fig. 8. Correlación entre IM y DV.

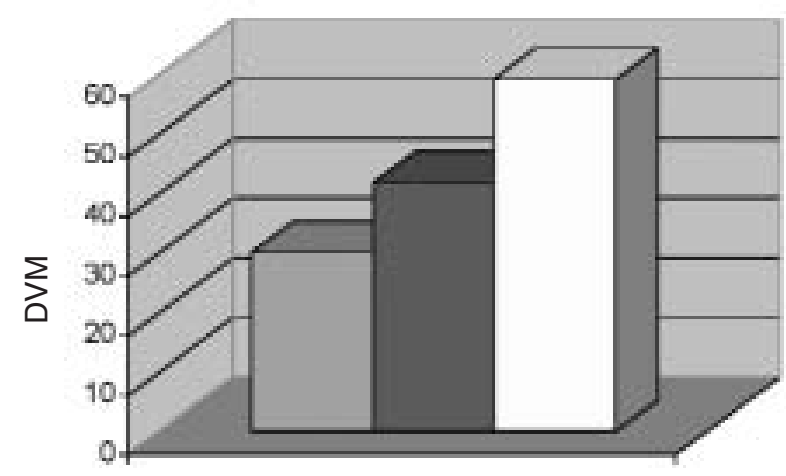

口GHI घGHII GH间

Fig. 9. Correlación entre GH y DV.

axilares metastáticos es sensiblemente mayor a la obtenida en los casos con ganglios no afectados (Fig. 11) y esta diferencia se mantiene cuando los

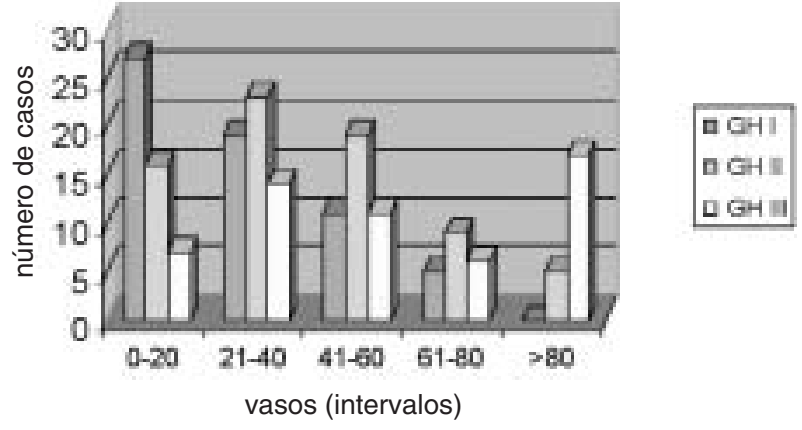

Fig. 10. Correlación entre GH y DV.

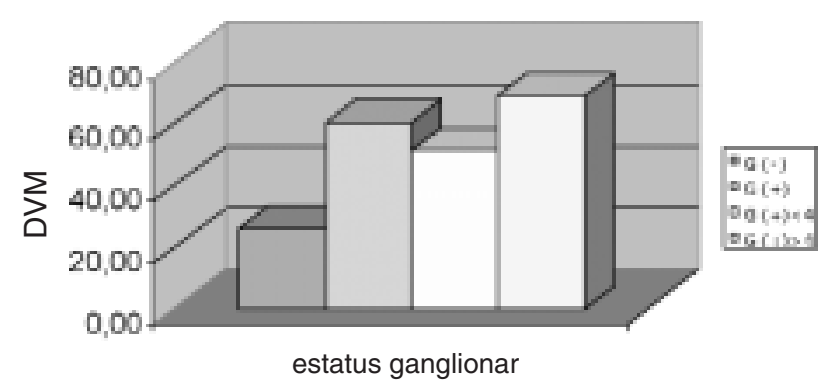

Fig. 11. Correlación entre EG y DV.

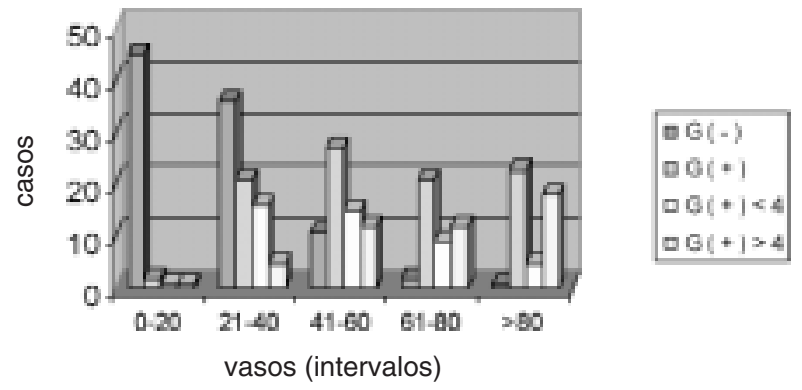

Fig. 12. Correlación entre EG y DV.

recuentos vasculares son agrupados en intervalos (Fig. 12).

Asimismo, se ha obtenido una DVM menor en los casos con menos de 4 ganglios afectados que en los casos con más de 4 ganglios metastáticos. Esta relación se mantiene al agrupar los recuentos vasculares por intervalos (Figs. 11 y 12).

\section{Discusión}

Diferentes parámetros histológicos han sido investigados, de forma singular o combinada, para determinar su valor pronóstico y/o predictivo en pa- 
cientes con cáncer de mama. El tamaño tumoral, estatus ganglionar axilar, grado histológico, grado nuclear, índice mitósico, tipo histológico y la expresión de receptores hormonales (estrógenos y progesterona) han demostrado su valor pronóstico y su utilidad clínica en el tratamiento de las pacientes ${ }^{52}$. A pesar de la variabilidad individual y de su heterogeneidad en cada tumor, la DV parece definirse como un factor pronóstico importante ${ }^{10,30,47,48}$ cuya utilidad ha sido valorada en distintos órganos y demostrada en el cáncer mamario y prostático ${ }^{33}$.

Varios autores niegan relación entre TT y DV $^{6,34,36-39}$. La correlación significativa entre estos dos parámetros establecida por otros $(18,48)$ es coincidente con nuestros resultados $(p=0,025)$. La correlación es más fiable cuando se establece mediante intervalos en el recuento vascular $(p=0,01)$.

Hemos encontrado correlación significativa entre la DV y los distintos grados de FT $(\mathrm{p}=0,002)$ que se mantiene al establecer intervalos en el recuento vascular $(p=0,01)$. Esta relación es especialmente significativa cuando se compara la DV obtenida en FT1 con los grupos restantes. Las diferencias son menos significativas al comparar los grupos FT2 y FT3.

Asimismo existen diferencias significativas entre la DV encontrada en los diferentes GN $(p=0,000)$ e IM $(p=0,000)$. No hemos encontrado referencias en la literatura a la relación entre FT y GN con la DV. En ambos grupos se mantienen las diferencias significativas cuando los recuentos vasculares se agrupan por intervalos.

La correlación entre GH y DV es altamente significativa $(p=0,000)$ y se mantiene cuando se establecen intervalos en el recuento vascular. Algunos autores sólo encuentran correlación entre la DV y este parámetro, pero no con otros factores pronósti$\cos ^{40}$. En análisis de supervivencia con multivariables, la DV supera en valor predictivo al TT, IM y $\mathrm{EG}^{49}$. Otros autores sin embargo, niegan la correlación de la DV con cualquiera de los factores pronósticos clásicos ${ }^{34,36-39}$.

Diferentes trabajos encuentran diferencias significativas entre las DV obtenidas en pacientes sin afectación de ganglios axilares y con metástasis en los mismos ${ }^{6,18,24,50-52}$. Asimismo diversos autores encuentran una relación directa entre la DV obtenida en las áreas de mayor DV del tumor (puntos calientes) y el riesgo de metástasis ganglionar ${ }^{5,6,27,50-52}$ y algunos encuentran relación entre la DV y el número de ganglios afectados ${ }^{25}$. Otros sin embargo discrepan de estos resultados ${ }^{34,36-39}$. Nuestro estudio encuentra diferencias estadísticamente significativas $(\mathrm{p}=0,000)$ entre las DV obtenidas en las series con diferente EG. Estas diferencias se mantienen cuando se establece la comparación entre la DV de la serie con ganglios negativos y los grupos con diferente número de ganglios positivos. Estas diferencias se mantienen al aplicar intervalos al recuento vascu$\operatorname{lar}(\mathrm{p}=0,000)$. En el análisis de la DV por intervalos hemos observado un aumento progresivo del número de casos con ganglios positivos, paralelo al aumento de la DV. De forma similar el número de pacientes con ganglios negativos disminuye en razón inversa al aumento de la DV. En ambos casos el cambio de tendencia se establece de forma significativa a partir de los 40 vasos. Estos hallazgos son coincidentes con los de otros autores con diferente $\mathrm{DV}^{6,18,24,52}$. Aplicando este punto de corte al resto de parámetros correlacionados con la DV hemos comprobado que se mantiene la correlación previamente establecida entre ellos. Esta correlación no es obtenida por otros autores en relación al TT ni al GH' .

En resumen, hemos obtenido una correlación estadísticamente significativa entre la DV, TT, TTM, con cada uno de los parámetros que integran el grado histológico combinado, con el GH, con el EG y con el número de ganglios afectados.

Correspondencia:

Dr. F. J. Torres Gómez

Plaza de la Encarnación, 32 - p1, 2B

E-41003 Sevilla

javiertorresgomez@yahoo.es 


\section{Bibliografía}

1. Aonuma M, Saeki Y, Akimoto T y cols. Vascular endothelial growth factor overproduced by tumor cells acts predominantly as a potent angiogenic factor contributing to malignant progression. Int J Exp Pathol 1999; 80, 271-281.

2. Guinebretiere J. Angiogenesis and breast neoplasm. The pathologists point of view. Gynecol Obstet Fertil 2005; 33, 140-146.

3. Huang B. Roles of main pro and anti-angiogenic factors in tumor angiogenesis. World J Gastroenterol 2004; 10, 467470 .

4. Vartanian R, Weidner N. Correlation of intratumoral endothelial cell proliferation with microvessel density (tumor angiogenesis) and tumor cell proliferation in breast carcinoma. Am J Pathol 1994; 1444, 1188-1194.

5. Weidner N, Semple J, Welch W y cols. Tumor angiogenesis and metastasis correlation in invasive breast carcinoma. N Engl J Med 1991; 324, 1-8.

6. Weidner N. The relationship of tumor angiogenesis and metastasis with emphasis on invasive breast carcinoma. Adv Pathol Lab Med 1992; 5, 101-121.

7. Edel M, Harvey J, Papadimitriou J. Comparison of vascularity and angiogenesis in primary invasive mammary carcinomas and in their respective axillary lymph node metastasis. Clin Exp Metastasis 2000; 18 (8), 695-702.

8. Folkman J, Watson K, Ingber D y cols. Induction of angiogenesis during the transition from hyperplasia to neoplasia. Nature 1989; 339, 58-61.

9. Gasparini G. Clinical significance of determination of surrogate markers of angiogenesis in breast cancer. Crit Oncol Hematol 2001; 37, 97-114.

10. Guinebretiere J. Angiogenesis and breast neoplasm. The pathologists point of view. Gynecol Obstet Fertil 2005; 33, 140-146.

11. Rak J, Filmus J, Kerbel R. Reciprocal paracrine interactions between tumor cells and endothelial cells: the "angiogenic progression“ hypothesis. Eur J Cancer 1996; 32, 2438-2450.

12. Esserman L, Hylton N, George T y cols. Contrast-enhanced magnetic resonance imaging to assess tumor histopathology and angiogenesis in breast carcinoma. Breast 1999; 1, 13-21.

13. Heffelfinger S, Yassin R, Miller M y cols. Vascularity of proliferative breast disease and carcinoma in situ correlates with histological features. Clin Cancer Res 1996; 11, 1873-1878.

14. Santinelli A, Baccarini M, Colanzi P y cols. Microvessel quantification in intraductal and early invasive breast carcinoma. Analyt Quant Cytol Histol 2000; 2, 277-284.

15. Zolota V, Geroskosta A, Melachrinou M y cols . Microvessel density, proliferating activity,p53 and bcl-2 expression in in situ ductal carcinoma of the breast. Anticancer Res : $1999 ; 19: 3269-3274$.

16. Beliën J, Somi S, De Jong J y cols. Fully automated micro- vessel counting and hot spot selection by image processing of whole tumour sections in invasive breast cancer. J Clin Pathol 1999; 52, 184-192.

17. Fox S, Gatter K, Bicknell R y cols. Relationship of endothelial cell proliferation to tumor vascularity in human breast cancer. Cancer Res 1993; 18, 4161-4163.

18. Fox S, Gatter K, Harris AL. Tumor angiogenesis. J Pathol 1996; 179, 232-237.

19. Edel M, Robbins P, D-Antuono M y cols. Assement of endotelial cell proliferation in primary breast carcinoma and its association with axillary lymph node status. Breast 2000;9 (1), 28 - 34.

20. Fox S, Leek R, Weekes M y cols. Quantitation and prognostic value of breast cancer angiogenesis: comparison of microvessel density, Chalkey count and computer image analisis. J Pathol 1995; 177, 275-283.

21. Heimann R, Fergurson D, Powers C y cols. Angiogenesis as a predictor of long-term survival for patients with nodenegative breast cancer. Br J Biomed Sci 1998; 3, 214-220.

22. Martin L, Holcombe C, Green B y cols. Is a histological section representative of whole tumour vascularity in breast cancer?. Br J Cancer 1997; 1, 40-43.

23. Wolf B. Histological grading in carcinoma of breast. Br J Cancer 1966; 20, 36-40.

24. Jiang X, Huang X, Li J. The correlation between tumor angiogenesis and lymph node metastasis in primary breast carcinoma. Zhonghua Wai Ke Za Zhi 1997; 35, 583-585.

25. Laforga J, Aranda F. Angiogenic index: a new method for assessing microvascularity in breast carcinoma with possible prognostic implications. Breast J 2000; 6, 103-107.

26. Heimann R, Hellman S. Individual characterisation of the metastatic capacity of human breast carcinoma. Eur J Cancer 2000; 36, 1631-1639.

27. Ogawa Y, Chung Y, Nakata B y cols. Microvessel quantitation in invasive breast cancer by staining for factor VIIIrelated antigen. Br J Cancer 1995; 72, 1297-1301.

28. Toi M, Kashitani J, Tominaga T. Tumor angiogenesis is a independent prognostic indicator in primary breast carcinoma. Int J Cancer 1993; 55, 371-374.

29. Bossari S, Lee A, De Lellis R y cols. Microvessels quantitation and prognosis in invasive breast carcinoma. Hum Pathol 1992; 23, 755-761.

30. Erden O, Dursun A, Loskun U y cols. The pronostic value of p53 and c-erb2 expresion, proliferative activity and angiogenesis in node-negative breast carcinoma. Tumori 2005; 91, 46-52.

31. Srivastava A. The prognostic significance of tumor vascularity in intermediate-thicness $(0,76-4,0 \mathrm{~mm}$ thick) skin melanoma. Am J Pathol 1988; 133,419-423.

32. Frangou E, Lawson J, Kanthan R. Angiogenesis in male breast cancer. World J Surg Oncol 2005; 3, 16.

33. Sharma S, Sharma M, Sarkar C. Morphology of angiogenesis in human cancer: a conceptual overview, histopro- 
nostic perspective and significance of neoangiogenesis. Histopathology 2005; 46, 481 - 489.

34. Goulding H, Rashid N, Roberston J y cols. Assement of angiogenesis in breast cancer: an important factor in prognosis ?. Human Pathol 1995; 26, 1196-1200.

35. Khanuja P, Fregene T, Gimotty P y cols. Angiogenesis does not predict recurrence in patients with primary breast cancer. Proc Am Soc Clin Oncol 1993; 12, 67.

36. Mayer M, Seshadri R, Raymond W y cols. Tumor microvascularity has no independent prognostic significance for breast cancer. Pathology 1998; 2, 105 - 110.

37. Pendleton N, Pazouki S, Heerkense E y cols. Relationship between different measurements of vascularity and clinicopathological parameters in breast cancer. Anticancer Res 1998; 68, 4565 - 4568.

38. Raymond J. Tumor angiogenesis inhibitors: media and scientific aspects. Presse Med.1998; 27, 1221-1224.

39. Siitonen S, Haapasalo H, Rantala I y cols. Comparison of different immunohistochemical metods in the assement of angiogenesis lack of pronostic value in a group of 77 selected node-negative breast carcinoma. Mod Pathol 1995; $8,745-752$.

40. Van Hoef M, Knox W, Dhesi S y cols. Assement of tumor vascularity as prognostic factor in lymph node negative invasive breast cancer. Eur J Cancer 1993; 8, 1141 - 1145.

41. Hansen S, Grabau D, Rose C y cols. Angiogenesis in breast cancer : a comparative study of the observer variability of methods for determining microvessel density. Lab Invest 1998; 78, 1563-1573.

42. Colpaert C, Vermeulen P, Benoy I y cols. Inflammatory breast cancer shows angiogenesis with high endothelial proliferation rate and strong E-cadherin expression. Br J Cancer 2003; 88, 718-725.

43. Lee J, Kim H Jung J y cols. Correlation between angioge- nesis, apoptosis and cell proliferation in invasive ductal carcinoma of the breast and their relation to tumor behavior. Analyt Quant Cytol Histol 2001; 23, 161-168.

44. Arora R, Joshi K, Nijhawan R y cols. Angiogenesis as an independent prognostic indicador in node-negative breast cancer. Analyt Quant Cytol Histol 2002; 24, 228-233.

45. Magennis D. Angiogenesis: a new prognostic marker for breast cancer. Br J Biomed Sci 1998; 3, 214-220.

46. Torres Gómez FJ, Torres Olivera FJ. Estudio comparativo de diferentes métodos de valoración de la angiogénesis en el carcinoma ductal infiltrante de mama. Rev Esp Patol 2006; 39(2): 87 - 92.

47. De Jong J, Van Diese P, Baak J. Hot spot microvessel density and the mitotic activity index are strong additional prognostic indicators in invasive breast cancer. Histopathology 2000; 36, 306-312.

48. Elston C, Ellis I. Pathological prognostic factors in breast cancer. I. The value of histological grade in breast cancer; experience from a large study with long-term follow-up. Histopathology 1991, 403-410.

49. Fitzgibbons P, Page D, Weaver D y cols. Prognostic factors in breast cancer. College of American Pathologists Concensus Statement 1999. Arch Pathol Lab Med 2000; 124, 966-978.

50. Ahlgren J, Risberg B, Villman K y cols.: Angiogenesis in invasive breast carcinoma. A prospective study of tumor hetereogeneity. Eur J Cancer 2002; 38, 64-69.

51. Xiao J, Yu X, Xu X y cols. Relation between angiogenesis, fibrinolisis and invasión of metastasis in breast cancer. Zonghua Zhong Lin Za Zhi 2005; 27, 226 -228.

52. Shpitz B, Bomstein Y, Stemberg A y cols. Angiogenesis, p53 and c-erb2 immunoreactivity and clinicopathological features in male breast cancer. J Surg Oncol 2000; 75, 252-257. 